\section{SP1-24 PROFILE EPIDEMIC OF THE ELDERLY ASSISTED IN THE PUBLIC HOSPITAL IN MACEIó X FREQUENCY OF FALLS}

doi:10.1136/jech.2011.142976n.1

${ }^{1} \mathrm{C}$ Fonsêca, ${ }^{2} \mathrm{~A}$ Pedrosa, ${ }^{*} \mathrm{D}$ Melo, ${ }^{4} \mathrm{M}$ Fernandes, ${ }^{5} \mathrm{G}$ Teixeira. ${ }^{1}$ Fundação Faculdade de Medicina, Universidade de São Paulo, FFMUSP, São Paulo, São Paulo, Brazil; ${ }^{2}$ Faculdade de Medicina, Universidade Federal de Alagoas, FAMED/UFAL, Maceió, Alagoas, Brazil; ${ }^{3}$ Hospital Universitário Professor Alberto Antunes - HUPAA/UFAL, Maceió, Alagoas, Brazil; ${ }^{4}$ Centro de Ciências Biológicas e Saúde, Universidade Católica de Pernambuco, Recife, Pernambuco, Brazil; ${ }^{5}$ Universidade Estadual de Ciências da Saúde de Alagoas-UNCISAL, Maceió, Alagoas, Brazil

Introduction It is believed that the falls are one of the great problems of the geriatrics. They present serious consequences in the elderly's bio-psico-social ambit, being mortality cause in that age group.

Methods The present work has as objective to trace the elderly epidemic profile assisted in the national health clinic of geriatrics of the Hospital Universitário Professor Alberto Antunes-HUPAA correlating with the frequency of falls. A cataloguing of the data contained in the record of per-existent evaluation, tends as variables: sex, age group, independence for activities of the daily life, occurrence of falls and the place, amount of medication that it uses and instruction level.

Results and Conclusion Before the analysed variables it can be observed that most of the elderly was independent and they had tendency to falls, most of them happened in the elderly own Lar and that great part made use of at least four medications.

\section{SP1-25 CORONARY HEART DISEASE PREVALENCE AND CORRELATES IN A BRAZILIAN POPULATION LIVING IN RIBEIRãO PRETO, SãO PAULO, BRAZIL, 2007}

doi:10.1136/jech.2011.142976n.2

S A de Moraes, * I C M de Freitas. University of São Paulo, Ribeirão Preto, São Paulo, Brazil

Background The demographic-epidemiological transition and the increasing of cardiovascular risk factors warrant epidemiological studies to identify coronary heart disease (CHD) prevalence and correlates in the Brazilian population.

Objectives To identify the prevalence of CHD and correlates in adults from Ribeirão Preto, São Paulo, Brazil.

Methods Cross-sectional population-based epidemiological study using three-stage cluster sampling. The variability introduced in the third sampling fraction was corrected by attributing equal weights to the number of eligible units in each domicile, resulting in a weighted sample of 1532 participants aged 30 years or older. The design effect was 1.33. Rose Questionnaire and ECG tracings classified by Novacode criteria ( $\mathrm{Q}$ waves and ST-T abnormalities) were used to estimate CHD prevalences $(95 \% \mathrm{CI})$. To investigate correlates, crude and adjusted prevalence ratios (PR) according to Novacode were estimated by points and $95 \%$ CIs, using Poisson regression.

Results The age-adjusted prevalence was $12.52 \% \quad(10.11 \%$ to $15.39 \%$ ) or $26.32 \%$ (22.87\% to $30.10 \%$ ) using Rose Questionnaire or Novacode, respectively. The Rose sensitivity and specificity compared to Novacode were, respectively, $16.08 \%$ and $88.76 \%$, and the area under the curve $\mathrm{AUC}=0.52$ (0.49 to 0.55). Age-genderadjusted prevalence ratios in the final model were represented by "age" $\mathrm{PR}=1.02$ (1.01 to 1.03 ); "hypertension" $\mathrm{PR}=1.65$ (1.23 to 2.19); "waist/height ratio" $\mathrm{PR}=1.54$ (1.05 to $2.25-2^{\circ}$ tertile) and 2.02 (1.15 to $3.56-3^{\circ}$ tertile); "hospitalisation" $\mathrm{PR}=1.36$ (1.04 to 1.78$)$ and "moderate physical activity" $\mathrm{PR}=0.57$ (0.34 to 0.94$)$, compared to the reference category. Trend test for PR across waist/height ratio tertiles were statistically significant $(p<0.05)$.

Conclusion The results show high CHD prevalence in the study population as well as identified correlates liable to public intervention policies.

\section{SP1-26 NEIGHBOURHOODS AND CENTRAL OBESITY IN A BRAZILIAN POPULATION-BASED STUDY: A MULTILEVEL ANALYSIS}

doi:10.1136/jech.2011.142976n.3

I C M de Freitas, ${ }^{*}$ S A de Moraes. University of São Paulo, Ribeirão Preto, São Paulo, Brazil

Objectives To estimate conicity index (CI) means and correlates. Methods Cross-sectional population-based epidemiological study using three-stage cluster sampling. The variability introduced in the third sampling fraction was corrected by attributing equal weights to the number of eligible units in each domicile, resulting in a weighted sample of 2197 participants aged 30 years and older, living in the urban area of Ribeirão Preto, São Paulo, Brazil, in 2006. Multilevel linear regression model was fitted to estimate $\beta$ s (individual level) and intra-regions variance (ecological level) considering 81 census tracts nested in four neighbourhoods (central-south, west, east, and north), and the conicity index as the outcome.

Results The crude CI means were higher in male (1.25) than in female (1.18). In final model, age $(b=0.003)$, family history of stroke $(b=0.008)$, BMI $(b=0.005)$, number of medicines taken $(b=0.004)$, years of smoking $(b=0.001)$, alcohol dependence $(b=0.011)$, and diet for weight loss $(b=0.015)$ were positively associated with CI. Gender $(b=-0.07)$, healthcare $(b=-0.011)$, and consumption of MUFAs $(b=-0.001)$ were inversely associated. The fraction of variance due to regions $(\rho)$ was $14.1 \%$. In both gender, CI adjusted means were high for nutritional status, smoking, alcohol dependence, and consumption of MUFAs, mainly in north and east regions.

Conclusion The results depicted the contribution of the ecological level to the conicity index, pointing out the role of correlates liable to intervention, which should be taken into account in planning prevention strategies, even considering that Ribeirão Preto city has been classified in the upper levels of Human Development Index.

\section{SP1-27 METABOLIC SYNDROME IN SOUTH ASIAN IMMIGRANTS: MORE THAN LOW HDL REQUIRING AGGRESSIVE MANAGEMENT}

doi:10.1136/jech.2011.142976n.4

S Dodani, ${ }^{*}$ M Butler, J Vacek. University of Kansas Medical Center, Kansas City, USA

Background Aggressive clinical and public health interventions have resulted in significant reduction in coronary artery disease (CAD) worldwide. However, South Asian Immigrants (SAIs) exhibit the higher prevalence of $\mathrm{CAD}$ and its risk factors as compared with other ethnic populations. The main objective of the current study is to assess the prevalence of metabolic syndrome (MS), its association with high density Lipoprotein (HDL) function, Apo lipoprotein A-I (Apo A-I) polymorphisms, and sub-clinical CAD using common carotid intima-media thickness (CCA-IMT) as a surrogate marker.

Methods Community-based cross-sectional study on SAIs aged 35-65 years was conducted. Sub-clinical CAD was measured using CCA-IMT. Dysfunctional/pro-inflammatory (Dys-HDL) was determined using novel cell free assay and HDL inflammatory index. 